\title{
Connectivity, Information Jumps, and Market Stability: An Agent-Based Approach
}

\author{
Khaldoun Khashanah and Talal Alsulaiman \\ Financial Engineering, Stevens Institute of Technology, Hoboken, NJ, USA \\ Correspondence should be addressed to Khaldoun Khashanah; kkhashan@stevens.edu
}

Received 2 April 2017; Accepted 18 July 2017; Published 22 August 2017

Academic Editor: Benjamin M. Tabak

Copyright ( $) 2017$ Khaldoun Khashanah and Talal Alsulaiman. This is an open access article distributed under the Creative Commons Attribution License, which permits unrestricted use, distribution, and reproduction in any medium, provided the original work is properly cited.

\begin{abstract}
We propose a metamodel to assess simulated market stability by introducing information connectivity in an agent-based network. The market is occupied by heterogeneous agents with different behaviors, strategies, and information connectivity. A jump-diffusion process simulating events that may occur in the market is introduced. Agents information awareness varies along with agents propensity to respond to the information jump and jump size. A jump reshuffles market positions based on agents risk preferences determined by behavior and strategy. We examine the effect of information awareness on the volatility index of the simulated market in a scale-free market network. The analysis is performed by developing five experiments wherein the first one corresponds to systemic information ignorance state. Three experiments examine the role of hubs, normal agents, and hermits in the network when intermediate combinations of agent types have information awareness. The fifth experiment corresponds to the systemic information awareness with all agents being informed. The results show that the simulated market is driven to instability in a similar manner to patterns observed in a crisis where all agents become homogeneous in information awareness of events. Hubs contribute to increased connectivity and act as amplifiers of good, bad, or inaccurate information or sentiment.
\end{abstract}

\section{Introduction}

How should a financial economic environment simulator (FEES) be designed and what should it inform? Our agentbased approach ultimately provides such a FEES just as a flight simulator offers the pilot a close-to-reality flying experience. However, the stage of reaching a truly complex financial environment simulator is not yet mature enough and this paper is one more step in that direction as it introduces information jumps, heterogeneous agent behavioral responses, information connectivity attributes, and a regulatory model in one financial network metamodel; the term metamodel in this paper is used in the sense that the ABM approach for a financial market network simulation constitutes a model of models. For the desired level of complexity, the financial economic dynamics cannot be, thus far, expressed analytically through a set of neatly justified equations. Thus, the advantage of agent-based metamodels lies in their ability to create sufficiently rich scenarios once the appropriate components of a financial system are included and endowed with their rules of interaction and transaction along with an initial state.

In this paper, we build on the previous work of Khashanah and Alsulaiman [1] where bounded rational heterogeneous market metamodel of network of networks was developed to explore the effect of the interaction of heterogeneous agents on the market volatility and capital. This paper develops the first metamodel for incorporating information jumps as shocks in an agent-based approach and to equip agents with heterogeneous response to information jump stimuli. Shocks are essential for meaningful financial systemic risk simulations. It is our philosophy to emphasize the quantitativesimulative-empirical approach, which aims to quantify the quantitative aspects of the problem, simulate the nonquantifiable aspects of the problem, and calibrate the metamodel parameters with empirical data. We do not agree with the 
excessive emphasis of some economic literature on analyzing systemic risk from the perspective of banking systems only. Markets, banks, intermediaries, media, and many other types of agents collectively interact as nonlinear dynamical systems in the financial ecosystem. Markets act as forward looking indicators of collective intelligence in all sectors including the banking system.

The novelty of our approach in this paper is to equip agents with new features representing their response to information flows including jumps. The idea that prices are information-driven was explained by many researchers starting with the work of Merton [2], yet those models assume that information has been factored into the price and may cause a jump in the price. Therefore, we obtain a jump-diffusion model depicting postulated price dynamics. However, market metamodels are interested in the mechanics by which the network supports the conversion of news into price impact. So far, market models in $\mathrm{ABM}$ have not included information sources as drivers of emerging patterns in market metamodels. As a first attempt to addressing this shortcoming, we observe that news are mappings of events into information from variable agent perspectives. Information packets flow in the network wherever there is a channel that supports that flow. Therefore, agents connectivity in our network refers to the ability to conduct information between two nodes. Thus we call it information connectivity. Since events map into information and events come in bursts of variable sizes, it makes sense to model information flow in a financial network as a stochastic jump-diffusion process that accounts for market events. Agents become receivers of information flows. To equip agents with this feature, we introduce the idea of agent information awareness in the network. Agents awareness variability of events and their variability of mapping events into actionable information both impact financial systemic patterns appearing in the financial system. In general, agents have heterogeneous information awareness; otherwise, agents are said to be homogeneous in information awareness.

In $\mathrm{ABM}$ we calibrate the model to either theoretical finance or empirical observations and then explore alternative scenarios that describe the relationship between macrobehaviors and micromotivations via the change of market microstructure. This relationship may be explored to produce the systemic patterns of markets under various scenarios. In this study, we take the maximum volatility index as a proxy measure of the systemic stability of the simulated market.

For the paper to be manageable, the analysis is carried out by developing five scenarios for agents information awareness of market events. The first and last scenarios are extreme cases where it is assumed that none of the agents is informed and, in the other, all agents are uniformly informed of the events. The second scenario assumes that the hub agents in the network are aware of events and they pass this information to the agents that are directly connected to them. Similarly, the third and fourth scenarios assume that agents with normal connections and hermits are aware of information jumps in the market, respectively.

The outline of this paper is as follows: Section 2 offers a literature review. Section 3 presents definitions of the market metamodel, models, and rules of interactions. Section 4 extends the connectivity model and Section 5 shows the simulation results and analysis of the experiments. We conclude with Section 6.

\section{Literature Review}

Researchers attempted to discover the causes of the anomalies in the stock market even before the development of the ABM. For over two decades researchers used ABM to explore complexities of a financial market. This includes the pioneering work of Kim and Markowitz [3] in 1989, the zero-intelligence model of Gode and Sunder [4] in 1993, the heterogeneous belief system of Brock and Hommes [5] in 1998, the Santa Fe artificial stock market [6] in 1999, and the Ising Model by Iori [7] in 2002.

Rules of interactions among market agents often find its roots outside the ABM literature. For example, Campbell and Kyle [8] derived an equilibrium model to examine the dynamics of stock prices. The model was constructed in a way that noise traders interact with smart money investors "fundamental traders." They conclude that "overreaction" to the news about the fundamental value creates an important type of noise to stock movements; the term "noise" has a different connotation than the one used in signal processing.

Existing models for analyzing financial systems are based on top-down approaches, as explained in Bookstaber [9], and focus on a comprehensive view while endeavoring to break down the system into components, which may result in losing a portion of variability such as behavior and agent interactions. Additionally, Bookstaber categorizes the applications of $\mathrm{ABM}$ in economics and finance into banks and assets management; real-estate markets; mortgage payments; payment systems and credit risk; financial market microstructure; and macroeconomics. In [10] Bookstaber et al. suggest that the new version of the stress test should require more emphasis on institutional interactions and feedback effects. Therefore, Bookstaber et al. recommend using more adaptable models, such as agent-based model, that are capable of capturing more elements of systemic complexity and can evolve freely from the history in a characteristic design similar to the real systems.

Sornette [11] called attention to utilizing models of complexity theory, such as network analysis and agent-based models (ABM) to assess systemic risk. Helbing [12] expressed that the catastrophe engendered by human factors can not be clarified by analytical approaches only but rather requires a collective comprehension of the social dynamics.

Thurner et al. [13, 14], as an implementation of ABM in banking and asset management, studied the effect of leverage on market behavior by building an ABM that allows borrowing long assets with a margin. The results of such actions cause fluctuations in prices, fat tail returns, and volatility clustering. As a mechanism for contagion, a bank may liquidate some of its assets to raise sufficient capital in response to a margin call. Asset liquidation to cover margin calls becomes a systemic risk when fire sale liquidations cause a downward spiral price destruction and eventual crashes in extreme cases. 
Poledna et al. [15] developed an ABM for simulating asset credit regulation policy. The assessments were performed under three different scenarios where in the first scenario the regulator imposes limits on maximum leverage only. In the second and third scenarios, Poledna et al. assessed the Basel II policy and alternative hypothetical ones by which the banks utilize options to hedge the risk completely. Kuzubaş et al. [16] simulate a financial network to examine the leverage effect in the banking system. The results indicate that the difference in leverage significantly affects the measure of systemic risk. The paper studied the impact of Basel III regulations for charging higher capital requirements for banks with higher leverage. The results confirmed that such a rule would increase the resilience of the system. In real-estate market application, Gilbert et al. [17] built up an ABM to research the housing market of the UK. The model accounts for interactions between the buyers, seller, and realtors given a set of endogenous and exogenous parameters representing agents attributes such as wealth and regulator control factors like interest rate. The results of the model show that house prices are sensitive to interactions of interest rate and loan-to-value (LTV) ratio. In addition, agents tend to cluster with high/low priced houses according to wealth. Also, altering the tax rate has an insignificant effect on house prices. Furthermore, a shock in the market causes fluctuations in house prices. Erlingsson et al. [18] generated an ABM of credit network and performed a set of simulation experiments to inspect the requirements for bank mortgages. The results show that lowering the mortgage standards increases house prices in the short term but leads to an unstable economic system in the long run and may lead to a recession. However, limiting home loans prerequisites helps market stability despite the possibility of slowing down economic growth. Other studies of systemic risk due to real-estate markets include the work of Carstensen [19], Bjarnason [20], and Ge [21].

Also, researchers addressed the role of network theory and agents direct interactions of model such as the models of Panchenko et al. [22], Yeh and Yang [23], Delellis et al. $[24,25]$, and Khashanah and Alsulaiman [1, 26]. For more details of the $\mathrm{ABM}$ in market microstructure, readers may refer to [27-30].

In postmodern finance with superconnectivity, agents in markets trade information dynamics. This paper is the first to introduce an information-based connectivity to model a financial network of market agent interactions, including information jumps, with heterogeneous agent response system to information flows using the ABM methodology. This paper investigates the impact of information connectivity on systemic volatility, which is proposed as an elementary proxy for the simulated market stability.

\section{The Metamodel and Models}

In this section, based on the metamodel of Khashanah and Alsulaiman [1,26], new definitions are introduced and the new features are explained. In the previous work the authors showed how the market volatility changes under heterogeneous agents behavior. In this paper we simulate financial market realizations to assess what may cause market instability as a function of information flow with jumps with heterogeneous agent information awareness and variable connectivity. For this purpose, we introduce the following definitions

Definition 1. The set $\mathscr{B}=\left\{B_{1}, B_{2}, \ldots, B_{m}\right\}$ denotes agent behavioral types; the set $\delta=\left\{S_{1}, S_{2}, \ldots, S_{k}\right\}$ denotes the set of $k$ strategies; and $\mathscr{D}=\{0,1, \ldots, n-1\}$ denotes a possible degree of an agent with $n$ the number of agents in the metamodel.

Definition 2. An agent is defined in terms of the attribute vector $\mathbf{a}^{(i)}=\left(b_{j}^{(i)}, s_{k}^{(i)}, d_{l}^{(i)}\right), 1 \leq i \leq n, b_{j}^{(i)} \in \mathscr{B}, s^{(i)} \in \mathcal{S}$, and $d_{l}^{(i)} \in \mathscr{D}$.

Definition 3. The agent lattice $\mathscr{A}$ is the set $\mathscr{A}=\mathscr{B} \times \mathcal{S} \times \mathscr{D}$.

Definition 4. A market $\mathscr{M}$ consists of an agent lattice $\mathscr{A}$, a set of assets $\mathscr{T}$, a set of regulatory frameworks $\mathscr{R}$, and a set of connectivity characteristics $\mathscr{C}$ summarized as the 4 -tuple $\mathscr{M}=(\mathscr{A}, \mathscr{T}, \mathscr{R}, \mathscr{C})$.

Definition 5. A market $\mathscr{M}$ is said to be homogeneous in behavior if agents exhibit one behavior so that $\mathscr{B}$ is a singleton; otherwise the market is said to be heterogeneous in behavior. A market $\mathscr{M}$ is said to be homogeneous in strategy if agents have one strategy so that $\delta$ is a singleton; otherwise, it is heterogeneous in strategy. A market $\mathscr{M}$ is said to be homogeneous in connectivity if agents are equally likely to be connected forming a random network; otherwise, $\mathscr{M}$ is said to be heterogeneous in connectivity.

Definition 6. A metamodel is a model of models. A market $\mathscr{M}$ is a metamodel consisting of its component models and their interactions expressed symbolically as the 4-tuple $\mathscr{M}=$ $(\mathscr{A}, \mathscr{T}, \mathscr{R}, \mathscr{C})$.

Current practices of scientific methodology assume linear interactions of models to produce a metamodel whose accuracy is as good as the minimum accuracy of its component models; this topic deserves attention as it has impact on advancing both complexity science and science complexity. A new definition of approximation and intermodel propagation of error is being developed in the context of quantitativesimulative-empirical methodology.

The metamodel is designed so that agents may interact directly in the trading environment where the network topology follows a scale-free network type that is structured based on the preferential attachment algorithm [31] with the initial number of hubs $H$. In the experiments of this paper, with Definitions 1 through 6, agents $\mathbf{a}^{(i)}, i=1,2, \ldots, 211$, with attribute vector $\mathbf{a}^{(i)} \equiv\left(b_{j}^{(i)}, s_{k}^{(i)}, d_{l}^{(i)}\right)$ with $k=1, \ldots, 4$, $j=1,2, \ldots, 6$, and $l=0,1, \ldots, 210$ referring to an agent degree. The behavioral types set is identified with $\mathscr{B}=$ $\{\mathrm{R}, \mathrm{RO}, \mathrm{RC}, \mathrm{L}, \mathrm{LO}, \mathrm{LC}\}$ where $\mathrm{R}, \mathrm{RO}, \mathrm{RC}$ stand for the risk averse, risk averse with overconfidence and risk averse with conservative types. In addition, L, LO, LC stand for the loss averse, loss averse with overconfidence and loss averse with conservative types. 
The strategies set, $\mathcal{S}$, consists of four strategies: the first strategy accounts for arbitrary traders that make investment decisions randomly, called zero-intelligence agents, and denoted by $Z$. The second investment strategy accounts for fundamental traders, denoted by $F$, who concentrate on the fundamental value of the asset, not on historical prices. The third type is referred to as momentum traders or technical traders, denoted by $T$, and the fourth type is called adaptive traders, denoted by $N$, with artificial intelligence capabilities using neural networks (NN); thus, $\mathcal{S}=\{Z, F, T, N\}$. The last two types distinguish themselves from the first two types in their practice that historical prices contain relevant information to future decisions.

Using Definition 5, the market $\mathscr{M}$ under consideration is heterogeneous in behavior, strategy and connectivity. The agent lattice $\mathscr{A}$ in this experiment is the collection of agents possible states. Thus the market $\mathscr{M}$ contains $|\mathscr{B} \times \mathcal{S}|=$ 24 possible types of agents as a result of combinations of behaviors and strategies. Allowing for degrees to change, we obtain the number of points in the agent lattice to be $|\mathscr{A}|=$ $24 \times 210=5040$, however, only 211 points in the agent lattice can be occupied at a given state of the market. Putting a practical upper limit on the agent degree reduces the agent lattice cardinality to reduce computational complexity.

The set of assets $\mathscr{T}$ in this experiment contains two assets: the risk-free rate asset and one risky asset. Furthermore, the regulatory set $\mathscr{R}=\left\{r_{f}, c, B, S\right\}$ with the risk-free rate $r_{f}$, taxation $c$, and buying and selling power $B$ and $S$ as control parameters. The regulatory environment can be designed to approximately map its real constructs in various financial economic systems.

The set of information connectivity characteristics depends on the network topology of the market. In this paper, our metamodel is heterogeneous in information connectivity in the sense of Definition 5 since the underlying network is scale-free. As part of connectivity characteristics, we introduce the new feature of heterogeneous ability to acquire information and be aware of information flows and call it information awareness in the market network. We consider this feature to be a function of agent connectivity and the coordinates of the agent in the agent lattice. More precisely, it is postulated that this property is a function of betweenness centrality of an agent. In our metamodel, heterogeneous agent awareness and the variability of mapping events into actionable information depending on agent awareness impact systemic patterns. The relationship between the interconnectivity and systemic risk depends on the definition of connectivity. In most studies on connectivity and systemic risk, connectivity is implied by the level of cash flow and risk flow, which are two important dimensions. In our context, information connectivity in terms of information flow is emphasized and, with third-party settlement, there is no direct transactional connectivity in this model. There is a reason for this type distinction between connectivity types: information dynamics, including market sentiments, lead to decisions on positions (such as buy, sell, or hold), which result in some transactions. In general, an information connectivity network is not identical to the corresponding transactional or cash flow connectivity network of the same market but rather the networks are dynamically linked with a variable lag. In an upcoming work, multidimensional connectivity types are considered including agent-bank, transactional, cash flow, and risk connectivity.

Agents belong to the market $\mathscr{M}$ to achieve their objectives. Agents myopically aim to maximize their utility function given the wealth constraint [32]. The solution of the optimization problem is given by

$$
x_{i, j, t}^{*}=\frac{E_{i, j, t}\left(p_{t+1}+d_{t+1}\right)-\left(1+r_{f}\right) p_{t} \pm c p_{t}}{\lambda_{i, j} v_{i, j} \beta_{i, j} \sigma_{i, t, p_{t+1}}^{2}+d_{t+1}},
$$

where $j$ denotes the behavior of the agent, which is identified with the set of behavioral types $\mathscr{B}$. With keeping the order, $\{\mathrm{R}, \mathrm{RO}, \mathrm{RC}, \mathrm{L}, \mathrm{LO}, \mathrm{LC}\}=\{1,2,3,4,5,6\}$ so that, for example, $j=1$ corresponds to risk averse type $\mathrm{R}$ and so on. The expectation $E_{i, j, t}\left(p_{t+1}+d_{t+1}\right)$ is the expected price and dividend for the next time step, which is crucial for the determination of optimal holding. The expectations of heterogeneous agents are by necessity diverse and they are determined based on investment strategies explained in the next section. Here $\sigma_{i, t, p_{t+1}+d_{t+1}}^{2}$ is the conditional standard deviation of price and dividend at time $t+1$. For simplicity, $\sigma_{i, t, p_{t+1}+d_{t+1}}^{2}$ is assumed to be constant of unit value. The change in the sign in the above equation opposite the state of $E_{i, j, t}\left(p_{t+1}+d_{t+1}\right)-\left(1+r_{f}\right) p_{t}$ makes $x_{i, j, t}^{*}=0$. By the change in the sign, we mean that the negative sign follows the positive state of $E_{i, j, t}\left(p_{t+1}+d_{t+1}\right)-$ $\left(1+r_{f}\right) p_{t}$ and the positive sign follows the negative state of $E_{i, j, t}\left(p_{t+1}+d_{t+1}\right)-\left(1+r_{f}\right) p_{t}$.

Agent behaviors are quantified by assigning values to coefficients. Hence, the risk aversion coefficient $\lambda=4$, loss aversion coefficient $\beta=2.5$ as $j=4,5$ or, 6 , and $\Delta W_{i, j, t}<$ 0 . Consider overconfidence/conservative $v$ where $v=v_{c}=3$ as $j=3$ or $6, v=1$ if $j=1$ or 4 , and $v=v_{o}=0.75$ as $j=2$ or 5 . The intuition behind the agent behaviors quantification is that the overconfidence/conservative agents tend to hold, sell higher, or lower portion of stock over the neutral traders. Also, the loss averse traders tend to reduce their positions as confronted with a loss at a given reference point $\Delta W_{i, j, t}$ as it has been indicated by Kahneman and Tversky [33]-individuals are impacted by losses more than profits.

The expectation $E_{i, j, t}\left(p_{t+1}+d_{t+1}\right)$ in (1) is a critical variable in the model. It is estimated individually given the agent trading strategy. The zero-intelligence agents quantitatively and randomly expect future prices and dividends as

$$
\widehat{E}_{i, j, t}=U\left(p_{0}-2 a, p_{0}+2 a\right)+U\left(d_{0}-2 b, d_{0}+2 b\right),
$$

where $a$ and $b$ are fixed at $a=270$ and $b=8$ in this study to reduce computational complexity and $U=U(a, b)$ is the uniform distribution on $[a, b]$ whose parameters can be calibrated.

The fundamental agents keep an eye on the fundamental process of the asset price where it is assumed that the dividends follow the process:

$$
d_{t}=d_{t-1} e^{\left(\mu_{d}-(1 / 2) \sigma_{t-1}^{2}\right) \Delta t+\sigma_{t-1} \sqrt{\Delta t} W_{t}},
$$


where $\mu_{d}=0.05$ is the growth rate of the dividend, $\sigma_{t}$ is the dividend volatility, and $W_{t}$ is a Wiener process with normal distributions $\sim N\left(0, \sigma_{W_{t}}\right)$. The variance $\sigma_{t}^{2}$ may be estimated using GARCH $(1,1)$ :

$$
\sigma_{t}^{2}=\alpha_{0}^{g}+\alpha_{1}^{g} r_{t-1}^{2}+\beta^{g} \sigma_{t-1}^{2},
$$

where $\alpha_{0}^{g}=0.0097, \alpha_{1}^{g} r_{t-1}^{2}=0.168$, and $\beta^{g} \sigma_{t-1}^{2}=0.766$. The fundamental prices of the asset then follow Williams [34]:

$$
p_{t}^{f}=\frac{d_{t}}{r_{f}}
$$

where $r_{f}$ is the risk-free rate. This fundamental value method works well as long as $0<r_{0} \leq r_{f}$.

The expected dividends and prices for agent $i$ are

$$
\begin{aligned}
\widehat{E}_{i, j, t}\left[p_{t+1}\right] & =\frac{\widehat{E}_{i, j, t}\left[d_{t+1}\right]}{r_{f}}, \\
\widehat{E}_{i, j, t}\left[p_{t+1}+d_{t+1}\right] & =\widehat{E}_{i, j, t}\left[p_{t+1}\right]+\widehat{E}_{i, j, t}\left[d_{t+1}\right] .
\end{aligned}
$$

The momentum agents verify the saying "the trend is your friend," and they buy/sell if the previous returns are positive/negative:

$$
\begin{aligned}
& \widehat{E}_{i, j, t}\left[d_{t+1}\right]= \begin{cases}d_{t}+d_{t}\left(\varphi_{i, j, t}\right) & \text { if } r_{d, t}>0 \\
d_{t}-d_{t}\left(\varphi_{i, j, t}\right) & \text { if } r_{d, t}<0 \\
d_{t} & \text { if } r_{d, t}=0,\end{cases} \\
& \widehat{E}_{i, j, t}\left[p_{t+1}\right]= \begin{cases}p_{t}+p_{t}\left(\varphi_{i, j, t}\right) & \text { if } r_{d, t}>0 \\
p_{t}-p_{t}\left(\varphi_{i, j, t}\right) & \text { if } r_{d, t}<0 \\
p_{t} & \text { if } r_{d, t}=0,\end{cases}
\end{aligned}
$$

where $\varphi_{i, j, t} \sim U(0.8,1.2)$.

The last trading strategy in $\mathcal{S}=\{Z, F, T, N\}$ in the strategy model is an adaptive trading strategy $N$ where the neural network is utilized for prediction execution. The designed neural network is a feed-forward network where the input to nodes $I N_{i, j}$ represents past returns of the stock that are uniformly distributed among the agents with the minimum of one previous return and maximum of ten past returns. Also, the neural network consists of one hidden layer that may be composed of one to ten nodes $H N_{i, j}$ with equal probability for agent $i$. The output of the neural network is the expected return at time $t+1$ that will be mapped to $\widehat{E}_{i, j, t}\left[p_{t+1}\right]$ and comparative approach applies to $\widehat{E}_{i, j, t}\left[d_{t+1}\right]$. Agents will learn by updating the weights inside the neural network from the training data $L$ where the training follows uniform distribution from 10 to 100 . The neural network is invoked customarily according to parameter $K=0.1$. The stopping criteria are subject to maximum iteration iter or base error $e$.

Agents may change their initial decisions of stock holding $x_{i}^{*}$ as a consequence of the interaction with other agents. Whenever agents have a direct interaction with each other with a chance to share their market sentiment, agents may be influenced to change their outlook on the market as a result of the interaction. Notice that market sentiment sharing is a form of information connectivity. The final holding decision $X_{i}^{*}$ is then constructed as the weighted average of the agent initial decision and initial decisions of the connected agents:

$$
\begin{aligned}
& X_{i, j, t}^{*} \\
& = \begin{cases}\alpha_{i, j} x_{i, j, t}^{*}+\frac{\left(1+\alpha_{i, j}\right)}{\sum_{k=1, k \neq i}^{N} I_{i k}} x_{i, j, t}^{*} & \text { if connections }>0 \\
x_{i, j, t}^{*} & \text { otherwise, }\end{cases}
\end{aligned}
$$

where $X_{i, j, t}^{*}$ is the final decision for agent $i$ and $\alpha$ is a given weight for the initial decision of holding shares of stock $x_{i, j, t}^{*}$ for agent $i, N$ is the total number of agents, and $I_{i k}=1$ if $k$ is connected to $i$ and zero otherwise.

The price formation will follow the price adjustment method [35-38]. The price adjustment process achieves local price equilibrium in the market based on the aggregate bids and offers

$$
p_{t}=p_{t-1}\left[1+\eta\left(B_{t}-O_{t}\right)\right],
$$

where $p_{t}$ is the market price at time $t, \eta$ is the price adjustment speed relative to the spread, that is, a simplified form of market efficiency. Further, $B_{t}$ represents the total number of bids among all agents and $O_{t}$ is the total number of offers.

\section{Exogenous Factors and Market Reactions}

In this study, information flow including jumps is introduced in the ABM metamodel. The market $\mathscr{M}$ "consumes" information and converts it through agent responses into new realizations of prices and positions. The market $\mathscr{M}$ may be influenced by nonsystematic events such as political, economic, and natural disasters, wars, or, in this day and age, tweets and fake news. For asset price dynamics, Merton's jump-diffusion model superposes jumps on a diffusion process [2] with jumps following a compound Poisson process. Merton's model is a special case of Lévy processes. Most literature in finance on jumps has been in conjunction with option pricing as the underlying asset undergoes a jump. A good reference on the subject of jumps is the book by Tankov [39]. For the purpose of this paper, and as a first approximation, we postulate that the information flow $I_{t}$ process follows a jump-diffusion process. The process of jumps follows the notation and construct in [40], with the usual definition of a probability space $(\Omega, \mathscr{F}, P)$ as a probability space with information filtration $\left\{\mathscr{F}_{t}\right\}_{t \geq 0}$ and the information process $I_{t}$ defined on the probability space with dynamics expressed by the stochastic differential equation (SDE)

$$
\frac{d I_{t}}{I_{t}}=\mu d t+\sigma d W_{t}+J(Q) d N_{t}
$$

where $\mu$ is the drift coefficients and $\sigma$ is the volatility-both are assumed to be constant. $W_{t}$ is a continuous Brownian 


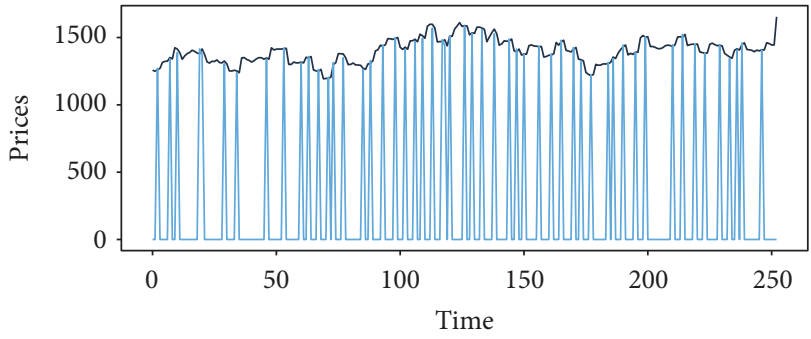

(a)

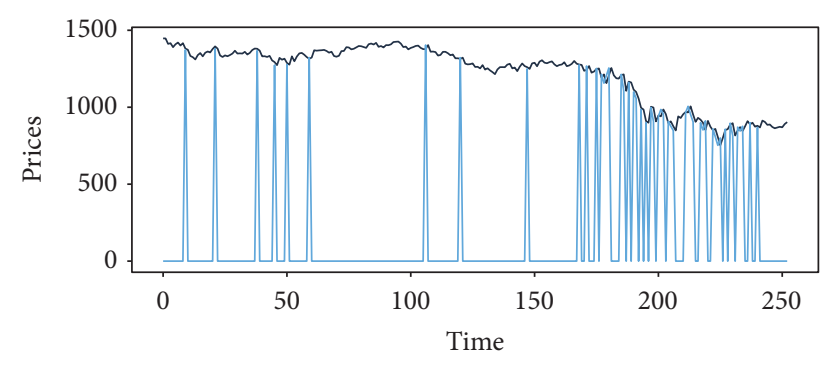

(b)

FIGURE 1: Simulated process $\lambda=0.2$ (a) and empirical jumps of 2008 S\&P 500 prices (b).

TABLE 1: Population size of agents types.

\begin{tabular}{lcccc}
\hline & $Z$ & $F$ & $M$ & \\
\hline Population size & & & & \\
R & 7 & 8 & 10 & \\
RO & 10 & 7 & 10 & 10 \\
RC & 7 & 8 & 10 & 9 \\
L & 8 & 9 & 7 & 9 \\
LO & 7 & 7 & 7 & 9 \\
LC & 11 & 14 & 8 \\
\hline
\end{tabular}

motion process and $N_{t}$ is Poisson process adapted to the filtration with constant rate $\lambda$ with $P\left(N_{t}=n\right)=\left((\lambda t)^{n} / n\right) e^{-\lambda t}$. As in [40], the term $J(Q)$ is a jump amplitude process. The three processes, $W_{t}, N_{t}$, and $J(Q)$, are assumed to be independent and the jump process, as in Merton model, is taken to be a compound Poisson process in the sense that we can sum jump amplitudes of Poisson jumps occurring over a given time interval. Using Itô calculus the solution of (10) takes the form

$$
I_{t}=I_{t-1} e^{\left(\mu-(1 / 2) \sigma^{2}\right) \Delta t+\sigma \sqrt{\Delta t} W_{t}+\mathrm{Q} \Delta N_{t}} .
$$

The price process is assumed to follow the same dynamics. Our implementation calibrates $\lambda$ to empirical jumps of price time series with a predefined jump threshold. For example, for the S\&P500 price process, for the year of 2008, with a threshold of $\pm 10 \%$, we collect return jumps in the set $J_{2008}\left\{r_{t_{k}}:\left|r_{t_{k}}\right|>0.1\right\}$, which is a time subseries of the return time series.

The jump size distribution, $Q$, can be assumed to be normally distributed which sufficiently achieves our implementation for this paper

$$
Q_{t}=\mu+\sigma \epsilon_{t}
$$

with constant $\widehat{\mu}$ being the jump mean and $\widehat{\sigma}$ being the jump standard deviation. Kou and Wang assume double exponential distribution as in "option pricing under a double exponential jump-diffusion model," Management Science, 50 (2004), pp. 1178-1192. For example, in the 2008 sample we find $\widehat{\mu}=-0.052$ for negative jumps and $\widehat{\mu}=0.045$ for positive jumps. The $\widehat{\sigma}=0.022$ is the jump standard deviation and $\epsilon_{t} \sim N(0,1)$.

Figure 1 shows the empirical jumps of the S\&P500 in 2008 (a) and the simulated process for the corresponding period with $\lambda=0.2$. The empirical jumps exhibit the phenomenon of jump clustering, which invites, in an upcoming work, the use of more sophisticated jump processes such as Hawkes selfexciting processes.

In this metamodel, the jump is restricted to being in response to an exogenous shock of the nonsystematic type as Merton [2] explained 'the Poisson distributed 'event' is an arrival of an important piece of information about the stock." However, the news arrival is converted by agents into actions of buy, sell, or hold, which collectively reflect in the jump price. In other words, the price jump is in fact an aggregate response to the stimuli of a jump in the information space. If the market is homogeneous in behavior, strategy, and connectivity, agents' response will be homogeneous.

Heterogeneous awareness of news produces variable time responses, so, as a first attempt, we relate the instances of jumps to agents' connectivity. There are a total of 211 agents in the trading space by which they remain distributed according to the behavior and strategy as shown in Table 1 .

The number of direct relations is varied among agents. The network degrees follow a power-law distribution with $H=5$. We divided agents in terms of connectivity into three classes: hubs, normal, and hermits. The links for each agent are demonstrated in Table 2. 


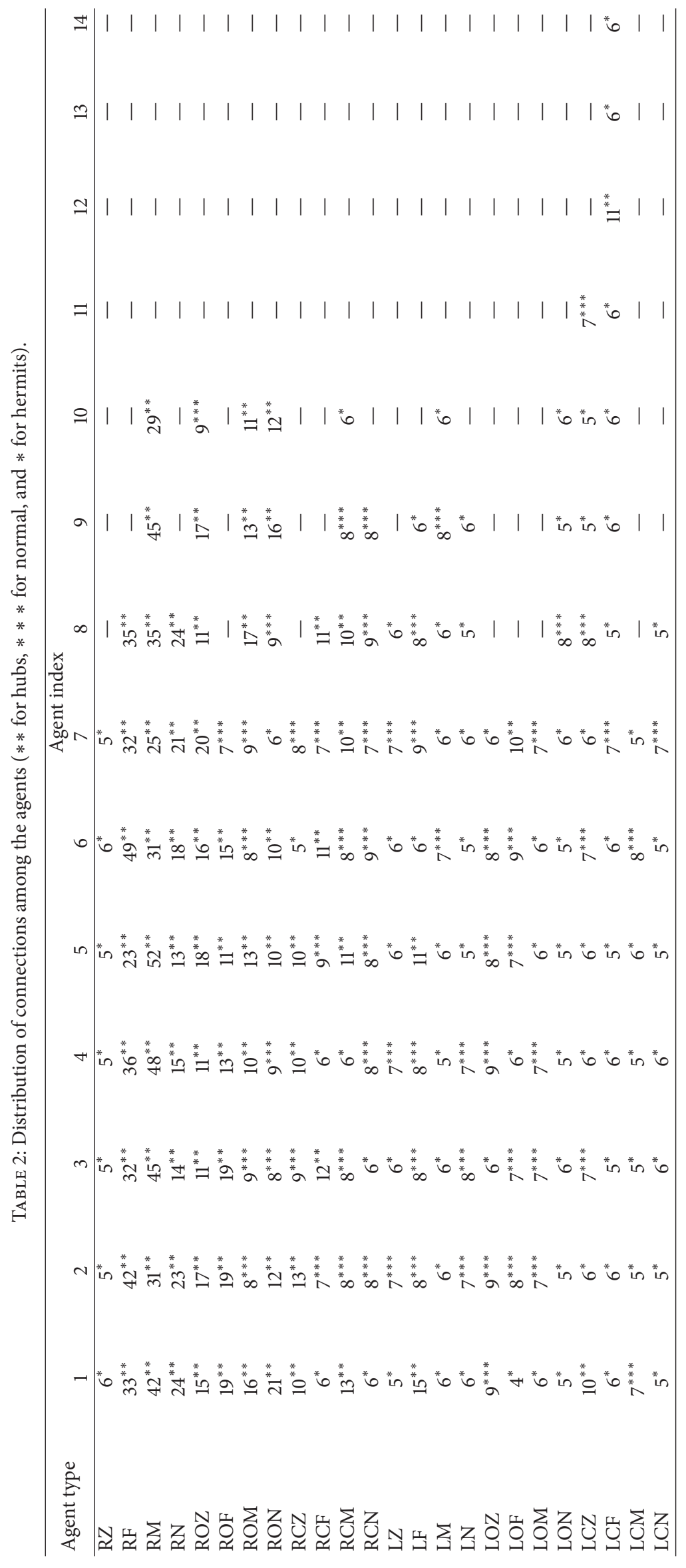


TABLE 3: Possible states of information flow awareness.

\begin{tabular}{lccccccc}
\hline Systemic ignorance & Only hubs & Only normal & Only hermits & $\begin{array}{c}\text { Normal and } \\
\text { hermits }\end{array}$ & $\begin{array}{c}\text { Hubs and } \\
\text { hermits }\end{array}$ & $\begin{array}{c}\text { Hubs and } \\
\text { normal }\end{array}$ & $\begin{array}{c}\text { Systemic } \\
\text { awareness }\end{array}$ \\
\hline 000 & 100 & 010 & 001 & 011 & 101 & 110 & 111 \\
\hline
\end{tabular}

The market's environment contains 69 individuals that have more than 9 connections. Also, it accommodates 60 agents with degrees between 7 and 8 and 82 agents with less than or equal to 6 edges. In addition, hubs connect with 1189 links, the normal class include 661 links, and hermits needed 464 links.

Mechanically, in order to incorporate the impact of jumps, we assume that agents exposed to the news process will reverse their position to be in the direction of the jump for a period that is proportionate to the jump size. On the other hand, the impact of a jump fades away as time progresses; thus, the design of a jump impact time function $\tau_{t_{0}}$ can be expressed as

$$
\tau_{t_{0}}=|\omega J(Q)| T \text { if jump occurs at } t_{0},
$$

where $\omega \sim N(0.04,0.01)$ represents an agent propensity to react to a jump, $T$ stands for the simulation termination time, $J(Q)$ stands for the magnitude of the jump, and $\tau_{t_{0}}$ expresses the period of time an agent is impacted by the jump occurring at $t_{0}$. The impact is realized through the agent reversal of a position from long (short) to short (long) on a negative (positive) jump with reversal impact time lasting $\tau_{t_{0}}$. An agent does not change position if the direction of the jump is aligned with his/her position so that, in that case, $\tau_{t_{0}}=0$. In the design of this metamodel, the market clock is shared by all agents so that trades occur at uniform discrete times. Agents who reverse positions continue doing so in subsequent trades until the impact fades away, that is, for a period of time $t \in\left[t_{0}, \tau_{t_{0}}\right]$ or unless a new jump arrives, whichever comes first. When a new jump arrives, the clock $\tau_{t_{0}}$ is reset to the new jump occurrence. Therefore, the function $\tau_{t_{0}}$ can be viewed as the time horizon of the impact of a jump of size $J(Q)$ occurring at time $t_{0}$ on an agent with parameter $\omega$ over a time of simulation $T$. In further work we extend the time horizon impact function to be a function of behavior and strategy.

Therefore, an agent reallocates positions conditional on the agent awareness of the jump according to

$$
\begin{aligned}
& \left(x_{i, j, t_{0}}^{*} \mid a_{i} \text { is aware of the jump }\right) \\
& = \begin{cases}-x_{i, j, t_{0}}^{*} & \text { if } x_{i, j, t_{0}}^{*} \text { position is opposite to jump direction } \\
x_{i, j, t_{0}}^{*} & \text { otherwise. }\end{cases}
\end{aligned}
$$

\section{Information Awareness and the Volatility Index}

We examine the market $\mathscr{M}$ under different systemic exposures $g$ to the information flow. The index $g$ represents the state of agents awareness in the market and these states can be large or infinite in general. For the sake of this paper, we present a discretization of such states by assigning binary states to each type of agent as defined with attribute vectors $\mathbf{a}^{(i)}=\left(b_{j}^{(i)}, s_{k}^{(i)}, d_{l}^{(i)}\right), i, 1 \leq i \leq n$. In this experiment, with the agent degree $d_{l}^{(i)} \in\{4, \ldots, 49\}$, there are three types of agents: hubs, normal, and hermits whose states of awareness are designated as $g_{1}, g_{2}$, and $g_{3}$, respectively. In general the index of type awareness can be partially realized but to simplify we assume that the type is either aware or not aware of market information; thus the range of type awareness takes values in $g_{i} \in\{0,1\}, i=1,2,3$. This simplification yields eight possible systemic awareness states ranging between systemic ignorance corresponding to state 000 and systemic awareness in state 111; see Table 3 .

The objective is to test the effect of agents information awareness of events on the volatility and standard deviation of returns. Formally we state the following hypothesis:

$$
\begin{aligned}
& H_{0}: g_{1}=g_{2}=g_{3}=0, \\
& H_{1}: \text { at least one } g_{i} \neq 0 .
\end{aligned}
$$

To test the hypothesis, a one-factor factorial design is implemented where the factor is type awareness of events. The null hypothesis $H_{0}$ is systemic ignorance while the alternate $H_{1}$ represents that at least one agent type has information awareness. The test aims to specify if there is a significant difference between the model outputs of maximum volatility of returns. The hypothesis can be rewritten in terms of the maximum volatility $V^{\max }$ and standard deviation $\sigma_{r}$ of returns.

A Monte Carlo simulation is implemented in order to collect the necessary statistical outputs for the ANOVA. The experiment replicates the Monte Carlo runs thirty times for each factor for a time horizon of 1000 days. This brings the total collected daily prices to 120,000 . From theses collected time series, we have calculated the daily returns and the volatility index. Subsequently, the ANOVA procedure tests whether there is a significant difference between maximum volatility and the standard deviations of the returns in the predescribed cases. Figures 7-11 show the results of simulation experiments. Figure 2 shows the box plot of the obtained maximum volatility index and Figure 3 shows return standard deviations.

ANOVA shows that there is a significant difference between the experiments where the $p$ values were close to zero. Table 4 summarizes the results of the analysis. The Tukey range test clusters the experiments into three groups in terms of the standard deviations of returns. The first group contains the case of systemic ignorance of jump events with the mean of annual volatility of returns over thirty runs of 
TABle 4: ANOVA table.

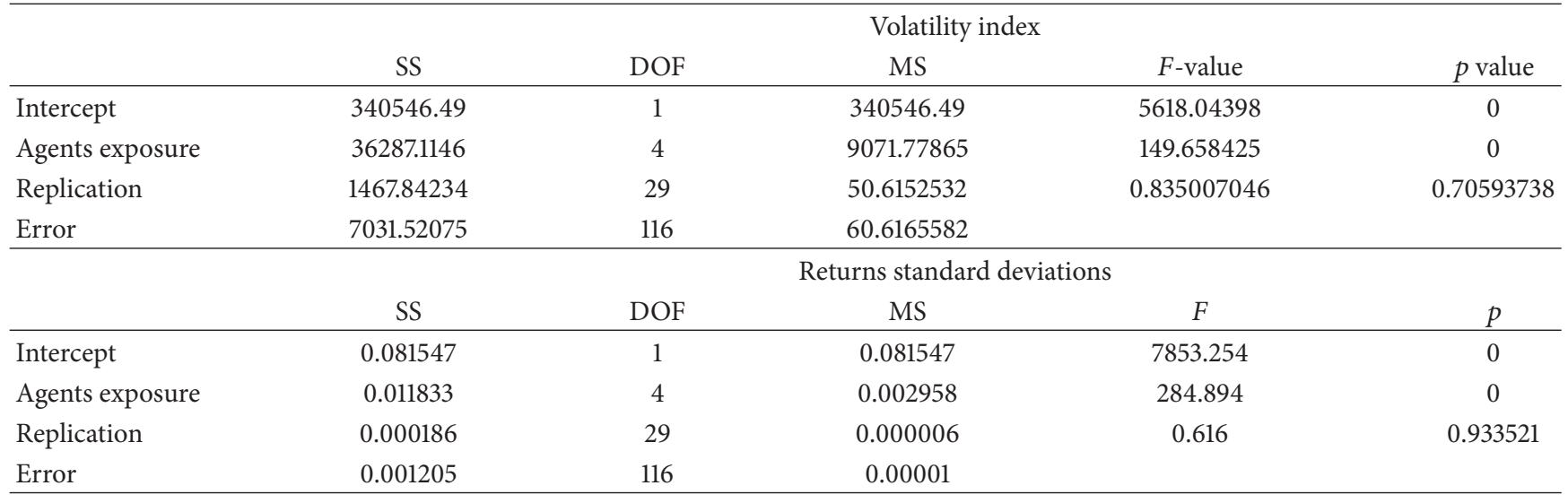

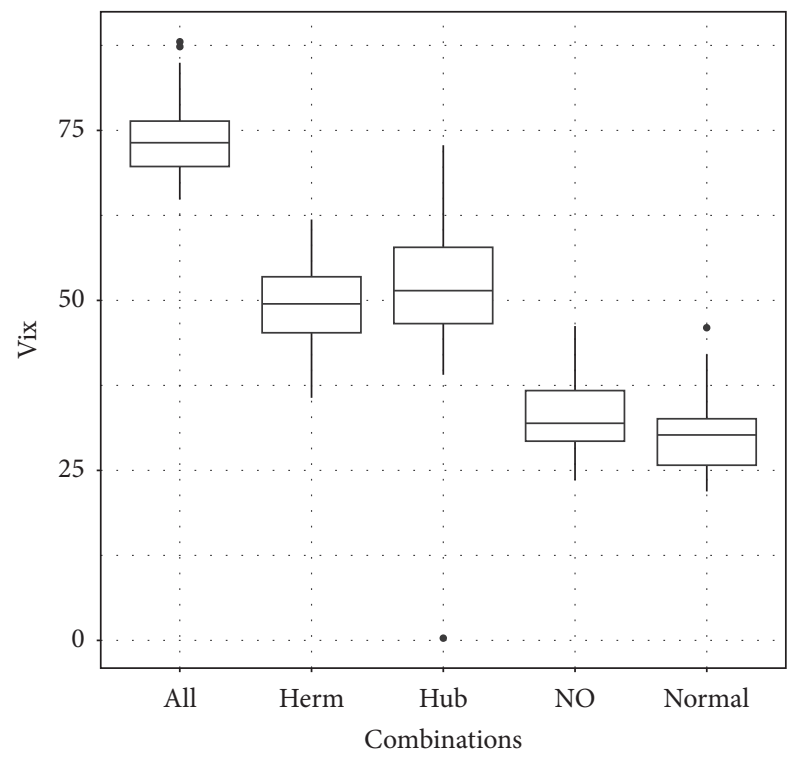

FIGURE 2: Box plot for the obtained simulation outputs of the maximum level of volatility index.

$12.5 \%$. The second group includes the group corresponding to the awareness state of 010 , that is, when only agents with normal connections are aware of events wherein the mean of the annual volatility of the thirty simulation runs was $29.8 \%$. The third group corresponds to state 111 of systemic awareness of jumps, which demonstrates that the annual volatility increases dramatically in a statistically significant manner compared to the other two states.

In terms of the volatility index, the experiments were classified into three groups as well. However, the results demonstrate that the maximum volatility index is significantly different from the other states when the agents are not aware of jumps or when only agents with normal range connection are exposed to the jumps. On the other hand,

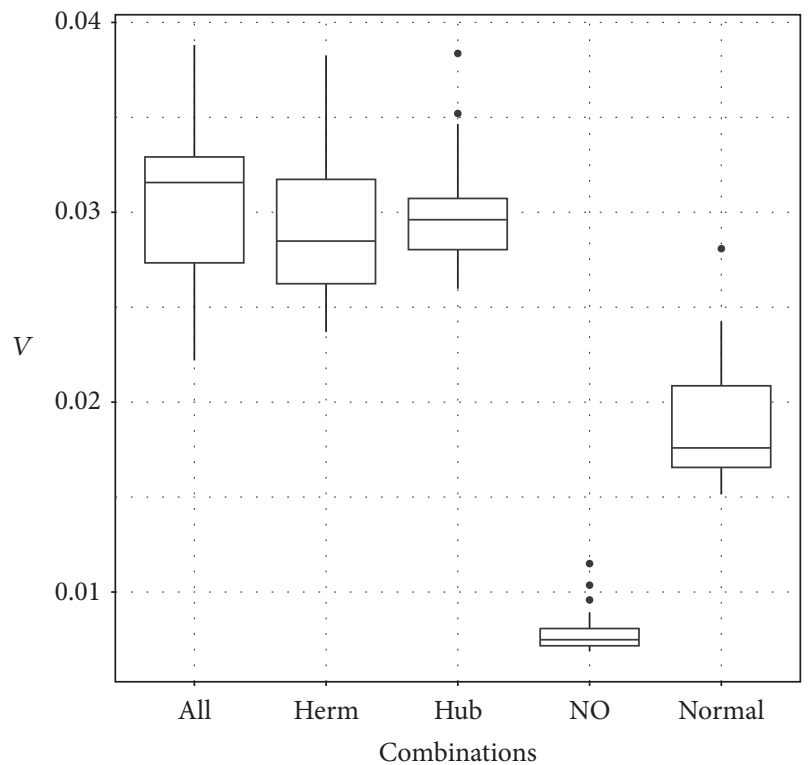

FIGURE 3: Box plot for the obtained simulation outputs of the mean returns standard deviation.

while no significant difference is observed on volatility of awareness between hubs (state 100) and hermits (state 001) agents to jump events in the market, both cases drive the simulated market out of stability. The average of maximum volatility index over the thirty runs realizes $51.1 \%$ and $49.2 \%$ for states 100 and 001 , respectively. Hubs have large connectivity and act as amplifiers of buy or a sell signal as they influence other agents by their decisions. Hubs increase the likelihood of information awareness convergence and in the extreme can lead to herding behavior. On the other hand, the population of hermits agents is quite large offsetting the perceived low number of connections (power of the masses). This property does not allow hermits to be influenced by the sentiment of other agents in the network 
and thereby their decisions cumulatively raise fear in the market.

The expected behavior of the market under modern finance axioms of no-arbitrage in a normal regime is similar to the state of systemic ignorance 000. The maximum observed volatility index was 31 for the year 2007 just before the financial crisis when the market shows a normal regime. However, in a rally regime such as that observed in the period between 2010 and 2014, the maximum volatility index was 48 . This behavior is similar to that when the hubs and hermits are aware of information. Furthermore, the overall behavior ranges between these cases when the normal agents are aware of the information.

Finally, in systemic awareness case 111, the fear level attains its volatility index maximum of 74.1 given all statistical tests. Empirically, in the presence of successive jumps and systemic awareness, as it was the case during the financial crisis of 2008, this result agrees simulatively with the empirical observation of VIX realizing its maximum of 80.86 on November 20, 2008.

Furthermore, we calculated the degree centralization of the network using Freeman's general formula [41]:

$$
C_{D}=\frac{\sum C_{D}\left(n^{*}\right)-C_{D}(i)}{[(N-1)(N-2)]}
$$

where $C_{D}$ represents the centralization of the network, $C_{D}\left(n^{*}\right)$ represents the maximum number of edges in the network, $C_{D}(i)$ represents the edges of node $i$, and $N$ represents the total number of nodes in the network.

The degree centrality of the calibrated network was 0.2 . Assuming that the volatility index can serve as a proxy measure of finical stability, we run Monte Carlo simulation of a decentralized network $\left(C_{D}=0\right)$. In the decentralized network, all agents are equally connected. Figures 4 and 5 show the calibrated and decentralized networks, respectively.

Under the assumption that $30 \%$ of the population has information awareness, the Monte Carlo simulation for thirty times is performed. The mean of the maximum volatility index records 23.5, which is close to the case of the normal agents awareness and systemic ignorance in the previous experiments. In addition, the mean of the return standard deviations of the runs is 0.014 , which is comparable to state 010. Figure 6 shows the market dynamics over the Monte Carlo runs.

In future research, we examine the market dynamics under various levels of network centralization. Also, we consider an assortative network structure wherein agents tend to share information with agents of similar attribute and compare it to a disassortative network structure where opposite-in-attributes may share information awareness.

The results can be partially compared to the model of Panchenko et al. [22] where they investigated the market under various network topology and concluded that the latency in information transmission (such as in lattice and small world network) may cause the market instability. However, the case here is quite different where our analysis is predicated on the market network following a power-law distribution and thereby allowing some nodes to be hubs with high degree and many hermits nodes with few edges. The main conclusion is that, in this simulated market, the aggressive reaction to the news from a large size of population or a population of highly connected individuals may drive the market out of stability.

\section{Conclusion}

The main contribution of this research is to advance a more realistic metamodel of markets that leads to a financial economic environment simulator. To this end, a formal mathematical definition of a market is introduced along with equipping agents with new attributes such as information awareness of jumps in the context of a scale-free network. This paper focuses on constructing a metamodel for stylized stability of simulated financial markets with emphasis on agents information connectivity, awareness, and propensity to respond to information exposure with a transaction. This paper offers a metamodel of a heterogeneous market where the agent transaction decisions are placed based on a certain set of attributes. Agents decisions change with behaviors, trading strategies, and the interactions with other agents. Trading strategies include arbitrary zero-intelligence, fundamental, trend following, and adaptive strategies that utilize neural network as a learning mechanism. In addition, behaviors such as risk and loss aversion, conservativeness, and overconfidence are included in the model. Also, agents interact directly by sharing market sentiment through a network following the power-law distribution.

A stochastic jump-diffusion process models jump events affecting the market. Agents may or may not be aware of these events depending on their classification. We introduce the concept of agent information awareness as an agent feature and make it a function of connectivity. If the event is known to the agent, the agent adjusts positions depending on the jump direction. The process will continue for a time-frame proportionate to the jump size, and thus we introduced the idea of a time horizon impact of a jump on an agent. Persistence of impact should be proportionate to the size of the jump and to agent propensity to react when a jump occurs.

Five experiments were designed to investigate the effect of agents' awareness of market dynamics and jump events. The analysis of the experiments was implemented using ANOVA. The results show that there is a significant dependence of outputs of various scenarios on the market information awareness states. The mean volatility and volatility index are lowest in the state of systemic ignorance. On the other hand, the volatility and fear index increase as hubs or hermits do observe jumps. The reason is that a large number of connections in the network belong to hubs, which increases the spread of news and impacts market sentiment collectively. The population size of hermits is quite large but relatively isolated from other agents and thus hermits are not influenced by market sentiment and this property can drive the simulated market out of stability. Normal agents information awareness has significant effects on market stability but does not reach the level of hubs and hermits 


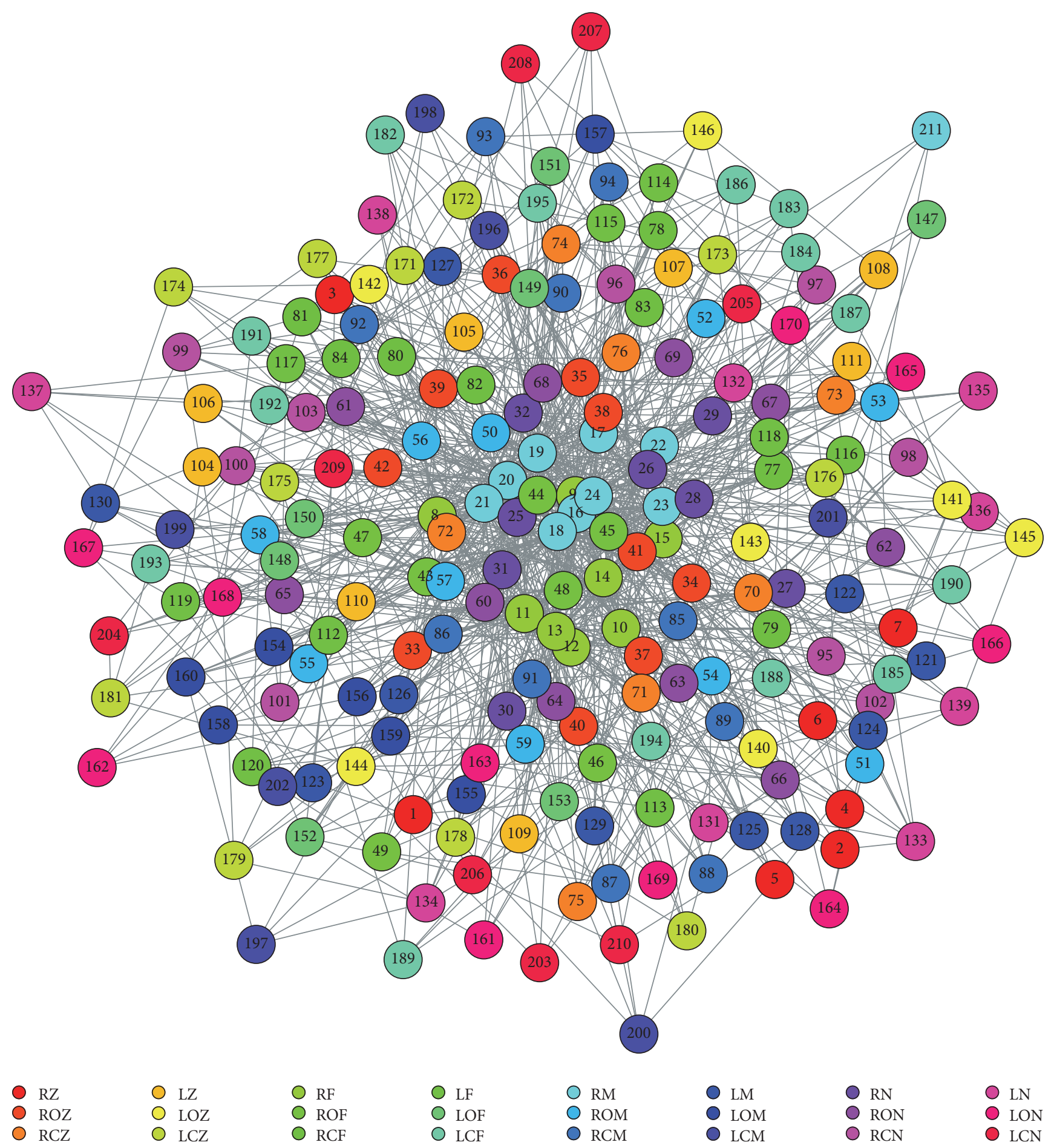

Figure 4: Calibrated network.

effects. In the case where agents information awareness of negative events increases, the fear index reaches its high limit.

In future research and in the context of financial economic environment simulator, we are interested in distinguishing between the state of persistent positive events that may lead to euphoric responses that ride on hopes of ever higher returns, on the one side, and negative large jumps causing excessive fear in agents and leading to the price destruction. It is postulated that both cases of excessive hope or fear exhibit information awareness convergence-one on the positive, optimistic side (false as it may be) and the other 


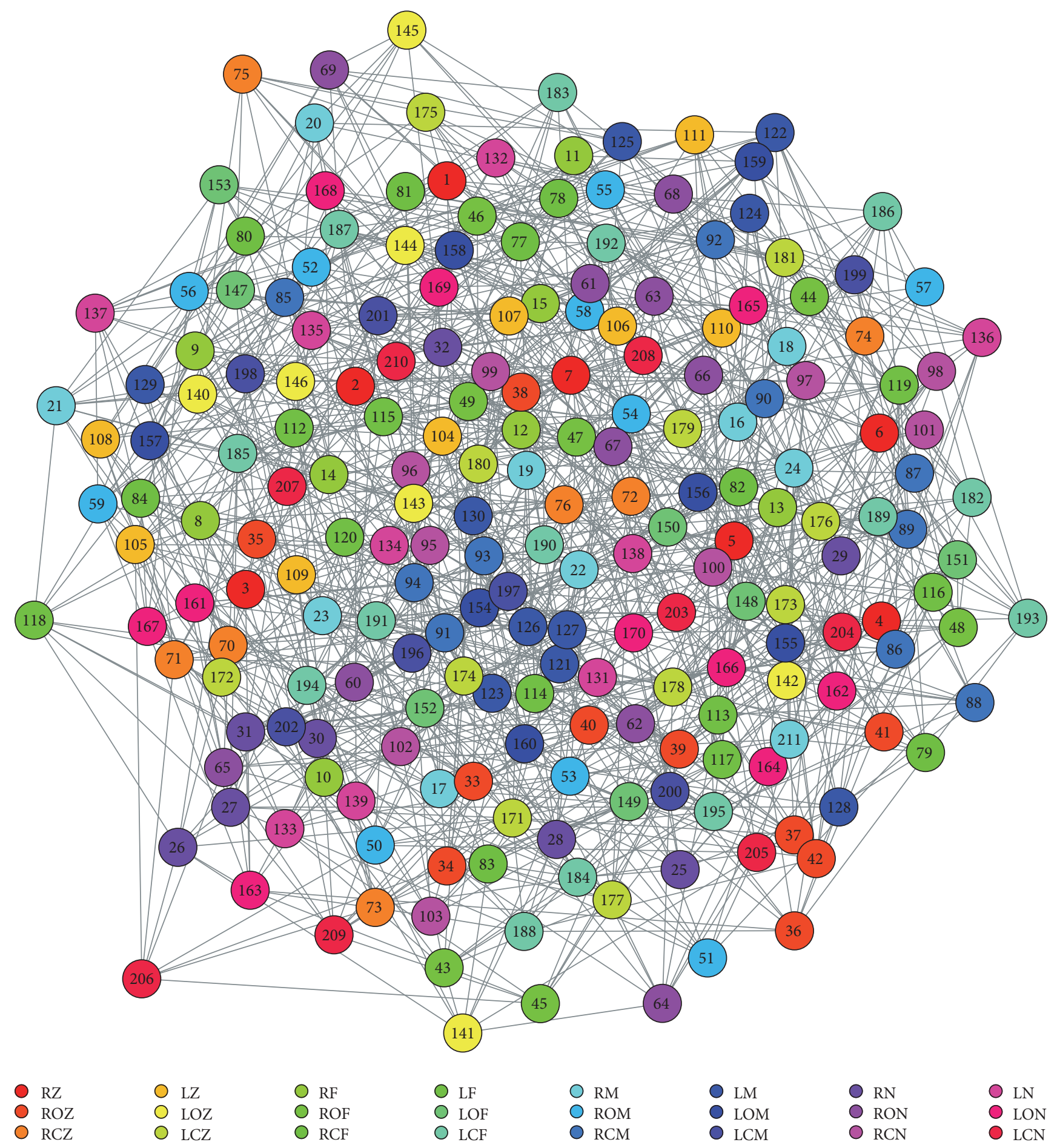

FIGURE 5: Decentralized network.

on the negative pessimistic side. In other words, a future financial economic environment simulator should be able to capture the state prior to the 2008 crisis wherein agents were in the state best described as "irrational exuberance" (Alan Greenspan) of unfounded hope of sustainable systemic overperformance and be able to model the opposite state of settling fear during a crisis where agents, individuals, institutional traders, and brokers converged to a negative homogeneous information awareness state, summarized by the market becoming to many as "uninvestable". 

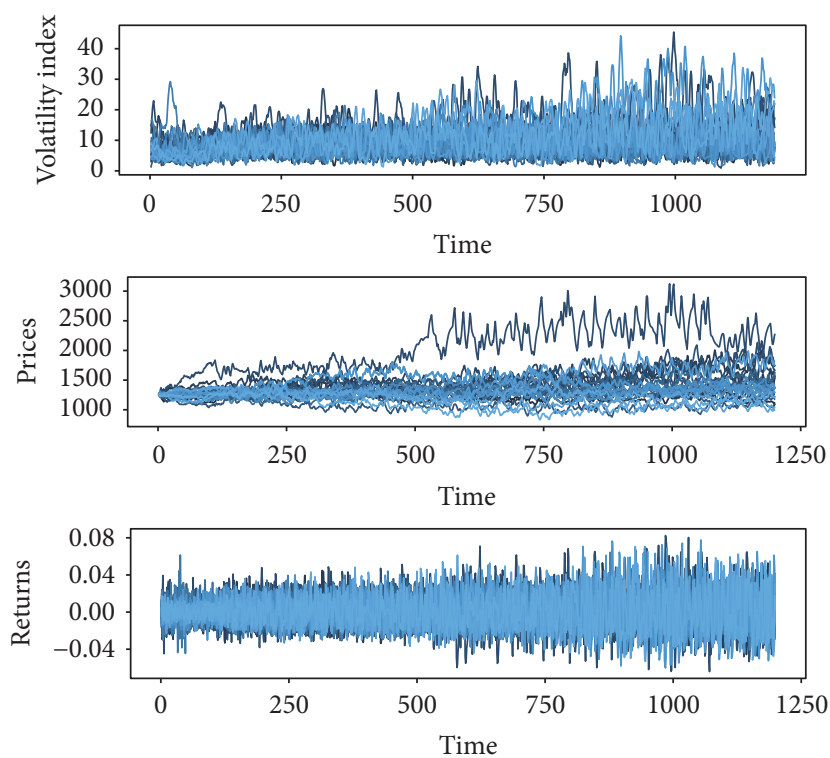

FIGURE 6: The market prices, returns, and volatility index under decentralized network.

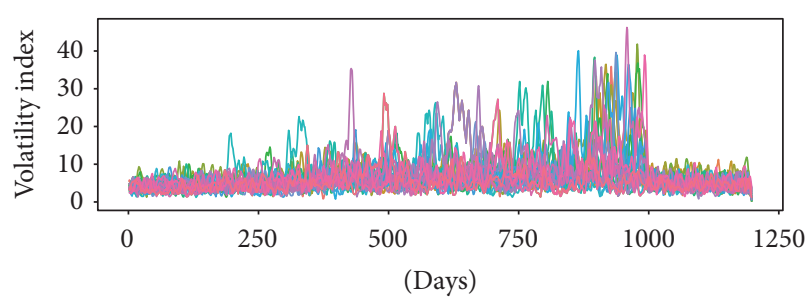

(a)

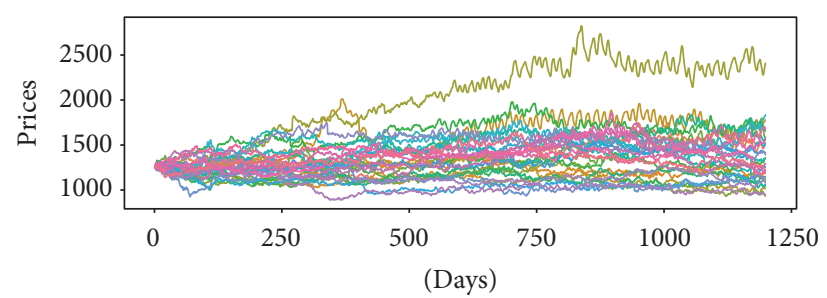

(b)

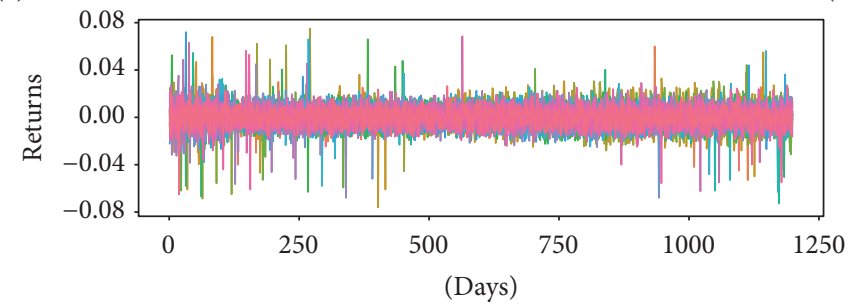

(c)

FIgURE 7: Time series of volatility index (a), prices (b), and returns (c), when $g=1=g=2=g=3=0$.

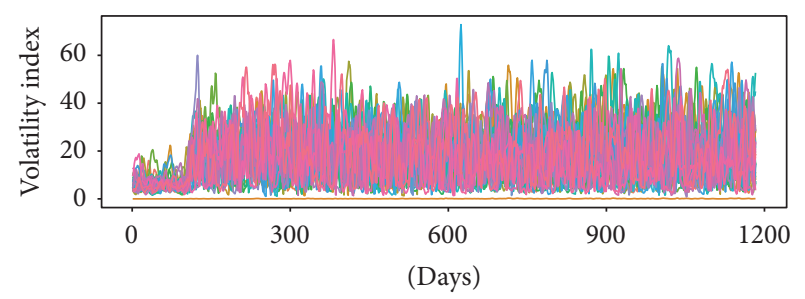

(a)

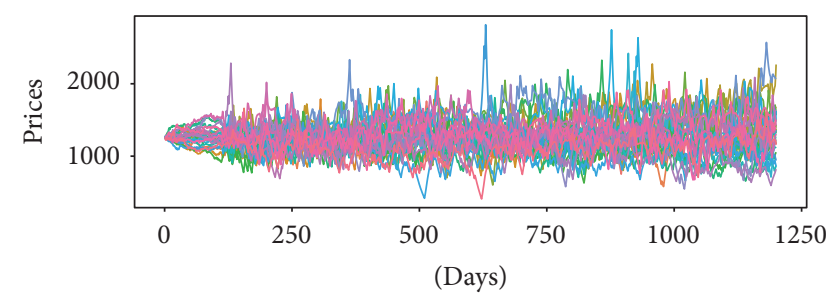

(b)

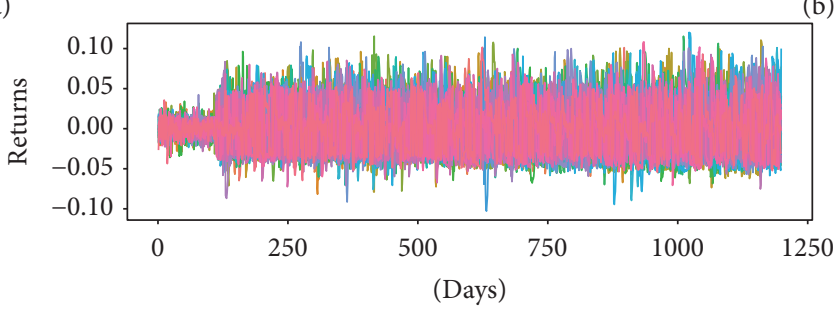

(c)

Figure 8: Time series of volatility index (a), prices (b), and returns (c), when $g=1=0$ and $g=2=g=3=0$. 


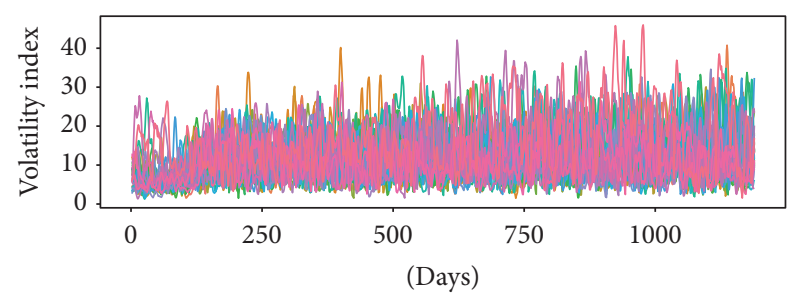

(a)

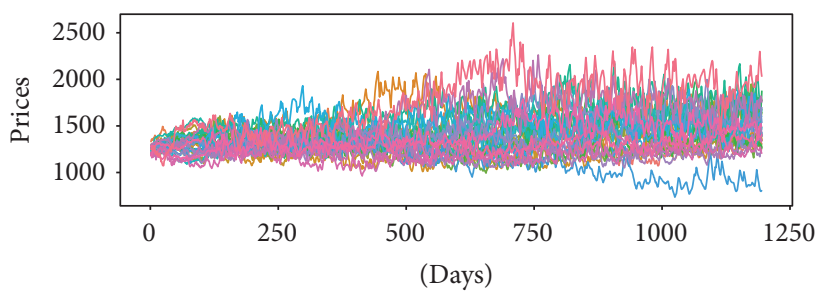

(b)

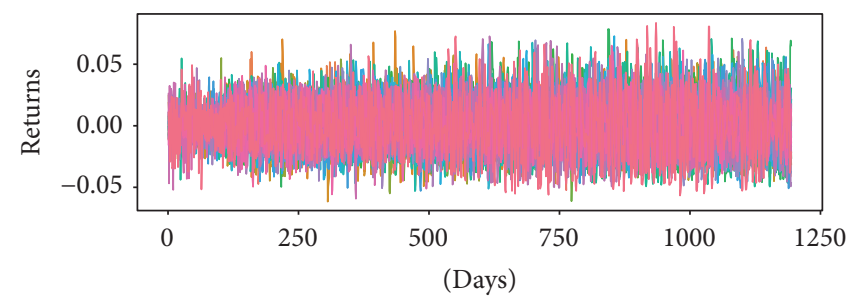

(c)

FIGURE 9: Time series of volatility index (a), prices (b), and returns (c) when $g=2=0$ and $g=1=g=3=0$.

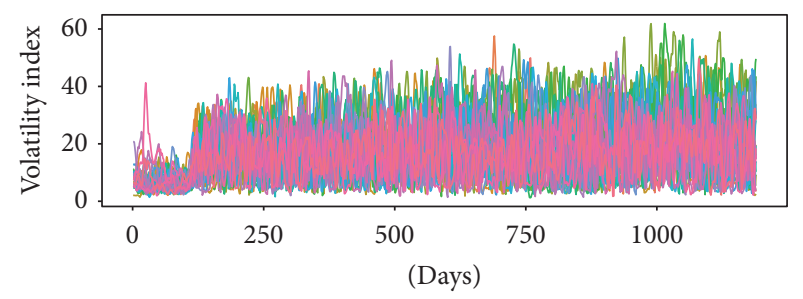

(a)

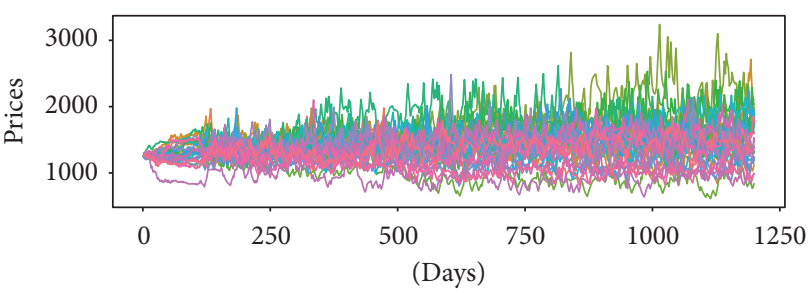

(b)

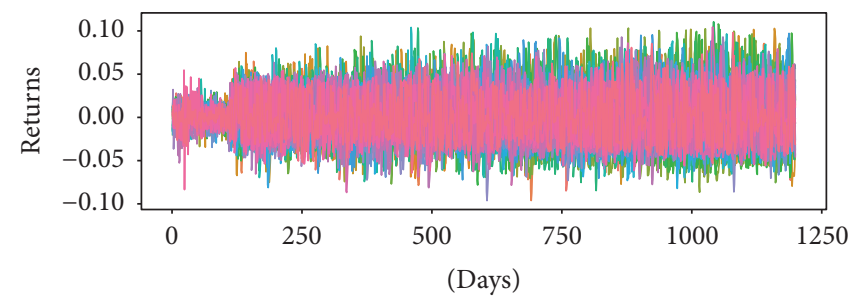

(c)

Figure 10: Time series of volatility index (a), prices (b), and returns (c) when $g=3=0$ and $g=1=g=2=0$.

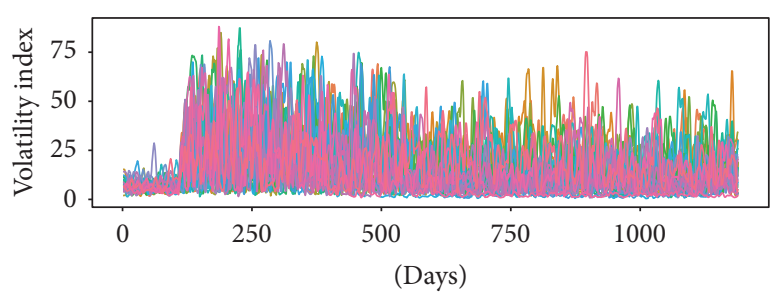

(a)

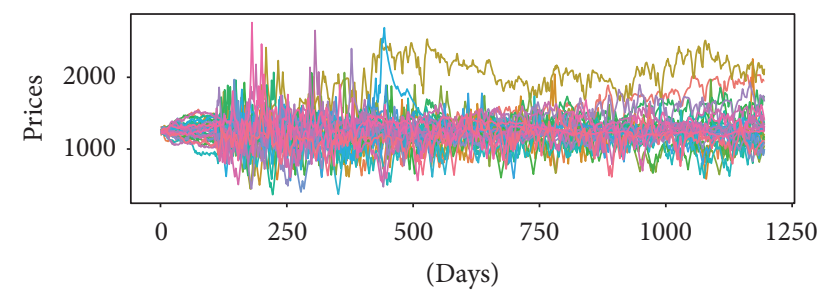

(b)

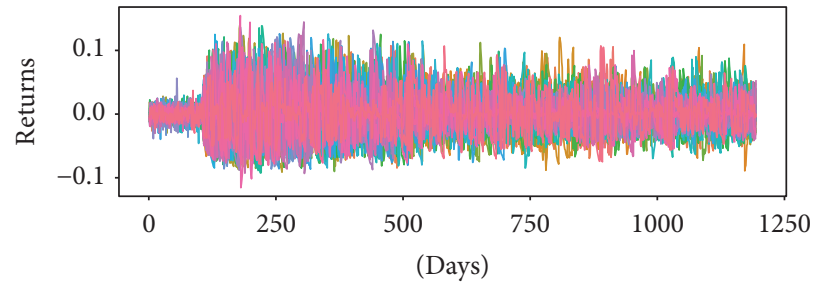

(c)

Figure 11: Time series of volatility index (a), prices (b), and returns (c), when $g=1=g=2=g=3=1$. 


\section{Conflicts of Interest}

The authors declare that they have no conflicts of interest.

\section{Acknowledgments}

The authors acknowledge the Hanlon Financial Systems Lab at Stevens Institute of Technology for providing the data and computational capabilities for this research work.

\section{References}

[1] K. Khashanah and T. Alsulaiman, "Network theory and behavioral finance in a heterogeneous market environment," Complexity, vol. 21, no. S2, pp. 530-554, 2016.

[2] R. C. Merton, "Option pricing when underlying stock returns are discontinuous," Journal of Financial Economics, vol. 3, no. 1-2, pp. 125-144, 1976.

[3] G. Kim and H. M. Markowitz, "Investment rules, margin, and market volatility," The Journal of Portfolio Management, vol. 16, no. 1, pp. 45-52, 1989.

[4] D. K. Gode and S. Sunder, "Allocative efficiency of markets with zero-intelligence traders: market as a partial substitute for individual rationality," Journal of Political Economy, vol. 101, no. 1, pp. 119-137, 1993.

[5] W. A. Brock and C. H. Hommes, "Heterogeneous beliefs and routes to chaos in a simple asset pricing model," Journal of Economic Dynamics and Control, vol. 22, no. 8-9, pp. 1235-1274, 1998.

[6] B. LeBaron, W. B. Arthur, and R. Palmer, "Time series properties of an artificial stock market," Journal of Economic Dynamics and Control, vol. 23, no. 9-10, pp. 1487-1516, 1999.

[7] G. Iori, "A microsimulation of traders activity in the stock market: The role of heterogeneity, agents' interactions and trade frictions," Journal of Economic Behavior and Organization, vol. 49, no. 2, pp. 269-285, 2002.

[8] J. Y. Campbell and A. S. Kyle, "Smart money noise trading and stock price behaviour," Review of Economic Studies, vol. 60, no. 1, pp. 1-34, 1993.

[9] R. Bookstaber, "Using Agent-Based Models for Analyzing Threats to Financial Stability," SSRN Electronic Journal, p. 24, 2012.

[10] R. Bookstaber, J. Cetina, G. Feldberg, M. Flood, and P. Glasserman, "Stress tests to promote finan-cial stability: Assessing progress and looking to the future," Journal of Risk Management in Financial Institutions, vol. 7, no. 1, pp. 16-25, 2014.

[11] D. Sornette, "Physics and financial economics (1776-2014): puzzles, Ising and agent-based models," Reports on Progress in Physics, vol. 77, no. 6, Article ID 062001, 062001, 28 pages, 2014.

[12] D. Helbing, "Systemic risks in society and economics," Understanding Complex Systems, pp. 261-284, 2012.

[13] S. Thurner, J. D. Farmer, and J. Geanakoplos, "Leverage causes fat tails and clustered volatility," Quantitative Finance, vol. 12, no. 5, pp. 695-707, 2012.

[14] S. Thurner, "Systemic financial risk: agent based models to understand the leverage cycle on national scales and its consequences," IFP/FGS Working Paper, vol. 14, 2011.

[15] S. Poledna, S. Thurner, J. D. Farmer, and J. Geanakoplos, "Leverage-induced systemic risk under Basle II and other credit risk policies," Journal of Banking and Finance, vol. 42, no. 1, pp. 199-212, 2014.

[16] T. U. Kuzubaş, B. Saltoğlu, and C. Sever, "Systemic risk and heterogeneous leverage in banking networks," Physica A: Statistical Mechanics and its Applications, vol. 462, pp. 358-375, 2016.

[17] N. Gilbert, J. C. Hawksworth, and P. A. Swinney, "Technosocial predictive analytics for security informatics," Security Informatics, pp. 30-35, 2009.

[18] E. J. Erlingsson, A. Teglio, S. Cincotti, H. Stefansson, J. T. Sturluson, and M. Raberto, "Housing market bubbles and business cycles in an agent-based credit economy," Economics, vol. 8, no. 1, 2014.

[19] C. L. Carstensen, An agent-based model of the housing market, [M.S. thesis], University of Copenhagen, Denmark, 2015.

[20] P. Bjarnason, Macroeconomic efiects of varied mortgage instruments studied using agent-based model simulations, [M.S. thesis], Reykjavík University, Iceland, 2015.

[21] J. Ge, "Endogenous rise and collapse of housing price: an agentbased model of the housing market," vol. 62, pp. 182-198, 2017.

[22] V. Panchenko, S. Gerasymchuk, and O. . Pavlov, "Asset price dynamics with heterogeneous beliefs and local network interactions," Journal of Economic Dynamics and Control, vol. 37, no. 12, pp. 2623-2642, 2013.

[23] C.-H. Yeh and C.-Y. Yang, "Social networks and asset price dynamics," IEEE Transactions on Evolutionary Computation, vol. 19, no. 3, pp. 387-399, 2015.

[24] P. Delellis, F. Garofalo, F. L. Iudice, and E. Napoletano, "Wealth distribution across communities of adaptive financial agents," New Journal of Physics, vol. 17, no. 8, Article ID 083003, 2015.

[25] P. DeLellis, A. DiMeglio, F. Garofalo, F. Lo Iudice, and C. Dovrolis, "The evolving cobweb of relations among partially rational investors," Plos One, vol. 12, no. 2, Article ID e0171891, 2017.

[26] K. Khashanah and T. Alsulaiman, "Heterogeneous financial markets as a network of networks," Computational Social Networks, 2017.

[27] C. H. Hommes, "Chapter 23 Heterogeneous Agent Models in Economics and Finance," Handbook of Computational Economics, vol. 2, pp. 1109-1186, 2006.

[28] B. LeBaron, "Chapter 24 Agent-based Computational Finance," Handbook of Computational Economics, vol. 2, pp. 1187-1233, 2006.

[29] S.-H. Chen, C.-L. Chang, and Y.-R. Du, "Agent-based economic models and econometrics," Knowledge Engineering Review, vol. 27, no. 2, pp. 187-219, 2012.

[30] T. Alsulaiman and K. Khashanah, "Bounded rational heterogeneous agents in artificial stock markets: Literature review and research direction," International Journal of Social, Behavioral, Educational, Economic and Management Engineering, vol. 9, pp. 2038-2057, 2015.

[31] A.-L. Barabfiasi and R. Albert, "Emergence of scaling in random networks," American Association for the Advancement of Science, vol. 286, no. 5439, pp. 509-512, 1999.

[32] S.-H. Chen and C.-C. Liao, "Price discovery in agent-based computational modeling of the artificial stock market," in Genetic Algorithms and Genetic Programming in Computational Finance, pp. 335-356, Springer US, Berlin, 2002.

[33] D. Kahneman and A. Tversky, "Prospect theory: an a nalysis of decision under risk," Econometrica: Journal of the Econometric society, vol. 47, no. 2, pp. 263-292, 1979. 
[34] J. B. Williams, The theory of investment value, 1938.

[35] M. A. Bertella, F. R. Pires, L. Feng, and H. E. Stanley, "Confidence and the stock market: an agent-based approach," PLos One, vol. 9, no. 1, Article ID e83488, 2014.

[36] R. G. Palmer, W. Brian Arthur, J. H. Holland, B. LeBaron, and P. Tayler, "Artificial economic life: a simple model of a stockmarket," Physica D: Nonlinear Phenomena, vol. 75, no. 13, pp. 264-274, 1994.

[37] J. Derveeuw, "Market dynamics and agents behaviors: a computational approach," in Artificial Economics, vol. 564, pp. 15-26, Springer, Berlin, 2006.

[38] S. Martinez-Jaramillo and E. P. K. Tsang, "An heterogeneous, endogenous and coevolutionary GP-based financial market," IEEE Transactions on Evolutionary Computation, vol. 13, no. 1, pp. 33-55, 2009.

[39] P. Tankov, Financial Modelling with Jump Processes, vol. 2, CRC press, Boca Raton, Fla, USA, 2003.

[40] D. Synowiec, "Jump-diffusion models with constant parameters for financial log-return processes," Computers and Mathematics with Applications, vol. 56, no. 8, pp. 2120-2127, 2008.

[41] L. C. Freeman, "Centrality in social networks conceptual clarification," Social Networks, vol. 1, no. 3, pp. 215-239, 1978-1979. 


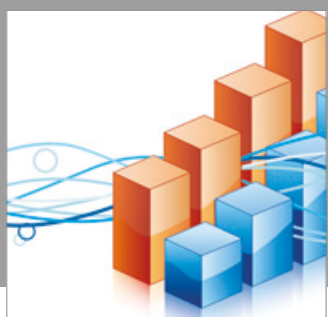

Advances in

Operations Research

vatersals

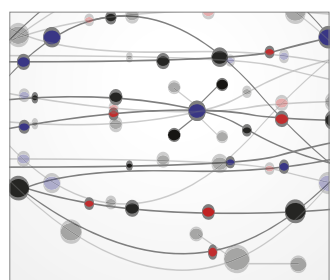

\section{The Scientific} World Journal
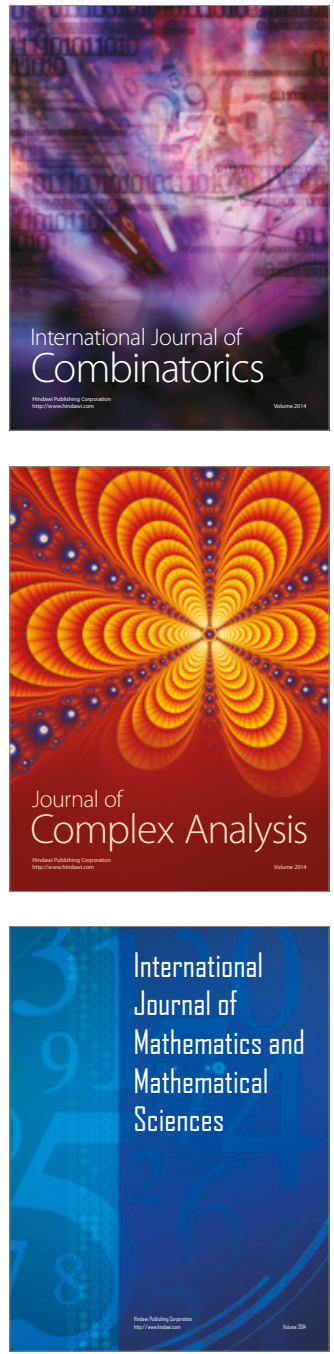
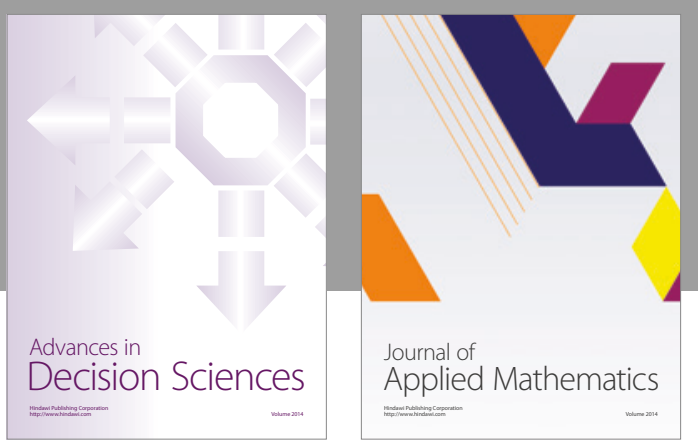

Algebra

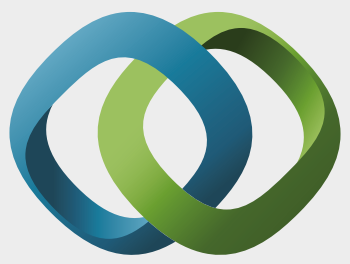

\section{Hindawi}

Submit your manuscripts at

https://www.hindawi.com
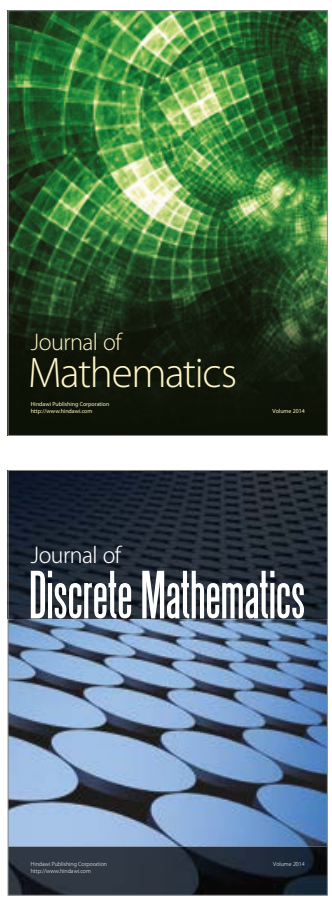

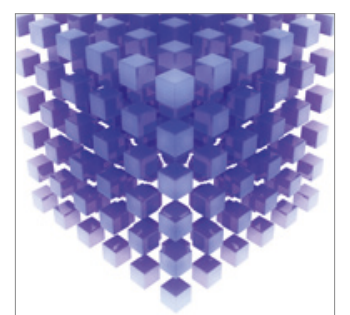

Mathematical Problems in Engineering
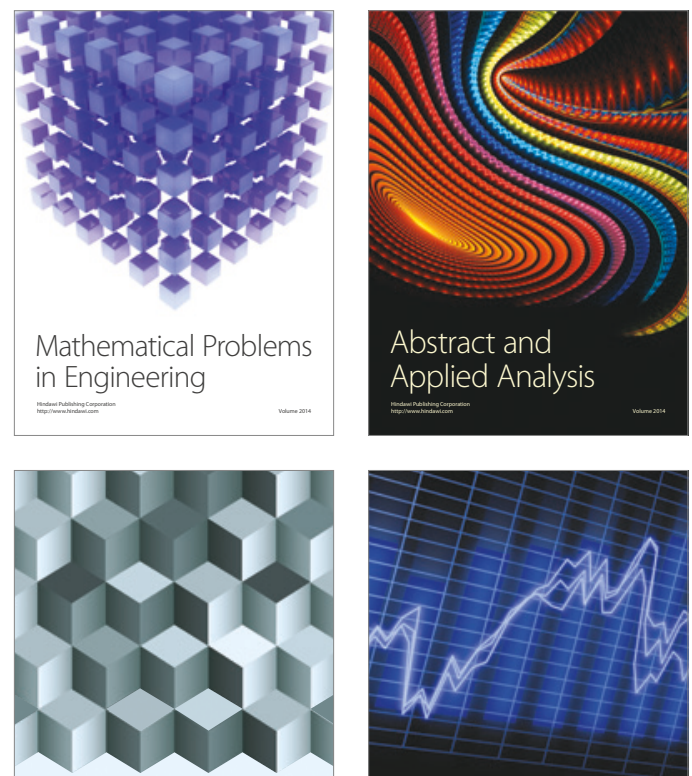

Journal of

Function Spaces

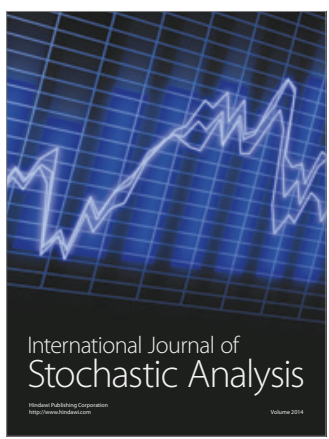

Probability and Statistics
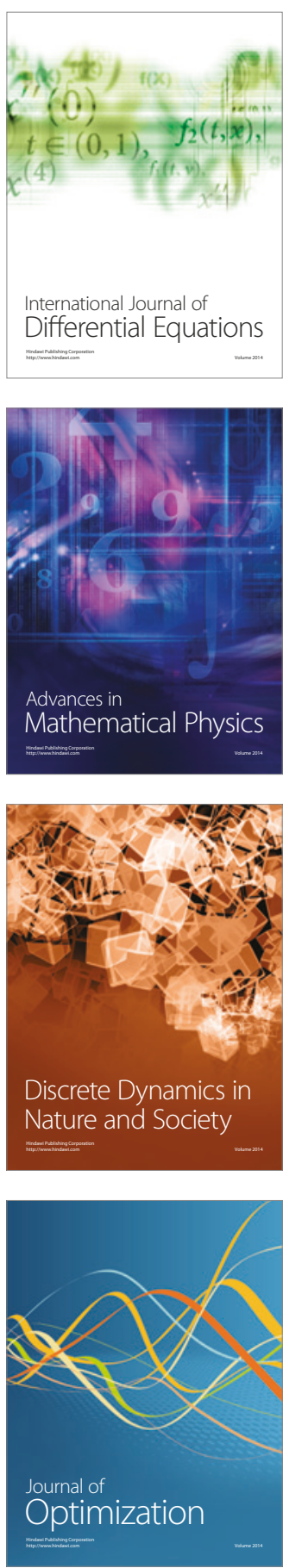